\title{
Progress Toward Polio Eradication — Worldwide, 2015-2016
}

\author{
Michelle Morales, MD ${ }^{1,2}$; Rudolf H. Tangermann, MD³; Steven G.F. Wassilak, MD²
}

In 1988, the World Health Assembly resolved to eradicate poliomyelitis. Wild poliovirus (WPV) transmission persists in only two countries (Afghanistan and Pakistan) after the removal of Nigeria from the list of countries with endemic polio in September 2015.* Indigenous WPV type 2 has not been detected since 1999 and was declared eradicated by the Global Commission for the Certification of Poliomyelitis Eradication in September 2015. ${ }^{\dagger}$ Since November 2012, when the last case of WPV type 3 was detected in Nigeria, WPV type 1 has been the sole circulating type of WPV (1). This report summarizes global progress toward polio eradication during 2015-2016 and updates previous reports (2). In 2015, $74 \mathrm{WPV}$ cases were reported in two countries (Afghanistan and Pakistan), a decrease of $79 \%$ from the 359 WPV cases reported in 2014 in nine countries; 12 WPV cases have been reported in 2016 (to date), compared with 23 during the same period in 2015 (3). Paralytic polio caused by circulating vaccine-derived poliovirus (cVDPV) remains a risk in areas with low oral poliovirus vaccine (OPV) coverage. Seven countries, including Pakistan, reported 32 cVDPV cases in 2015 (4). In four of these countries, $\geq 6$ months have passed since the most recent case or isolate. One country (Laos) with VDPV transmission in 2015 has reported three additional cVDPV cases in 2016 to date. Encouraging progress toward polio eradication has been made over the last year; however, interruption of WPV transmission will require focus on reaching and vaccinating every missed child through high quality supplementary immunization activities (SIAs) and cross-border coordination between Afghanistan and Pakistan $(5,6)$.

\section{Routine Poliovirus Vaccination Coverage}

Estimated global coverage among infants aged $\leq 1$ year with 3 doses of OPV (OPV3) through routine immunization was $88 \%$ in 2014 (the most recent year for which complete data are available). WHO and the United Nations Children's Fund estimate that OPV3 coverage by WHO region was 80\% in the African Region, $86 \%$ in the Eastern Mediterranean Region, 95\% in the European Region, 90\% in the Region of the Americas, 90\% in the South-East Asia Region, and 87\% in the Western Pacific Region; considerable inter- and intracountry variability exists. National OPV3 coverage was 75\% in Afghanistan and 72\% in Pakistan; coverage is estimated to be substantially lower in areas with WPV transmission (5-7).

\footnotetext{
*http://www.who.int/mediacentre/news/releases/2015/nigeria-polio/en/.

${ }^{\dagger}$ http://www.polioeradication.org/mediaroom/newsstories/Global-eradicationof-wild-poliovirus-type-2-declared/tabid/526/news/1289/Default.aspx.
}

\section{Supplementary Immunization Activities (SIAs)}

In 2015, approximately 2 billion OPV doses were administered during 231 SIAs in five WHO regions (Table 1), including 1.2 billion doses administered during national immunization days, 770 million during subnational immunization days, 11 million during child health days, and 22 million during large-scale door-to-door SIAs ("mop-up" activities) in areas where poliovirus was known or suspected to be circulating. Approximately 1.2 billion of the administered doses were trivalent (tOPV, containing OPV types 1, 2, and 3), 843 million were bivalent (bOPV, containing types 1 and 3 ), and 5 million were monovalent type 1 OPV doses.

\section{Poliovirus Surveillance}

Polio cases caused by either WPV or cVDPV are detected through surveillance for acute flaccid paralysis (AFP) and subsequent stool specimen testing at WHO-accredited laboratories in the Global Polio Laboratory Network. The main indicators of adequate surveillance include 1) an annual nonpolio AFP rate of $\geq 1$ case per 100,000 population aged $<15$ years for countries in $\mathrm{WHO}$ regions certified as polio free, or a rate of $\geq 2$ for all other countries and 2) adequate stool specimens collected from $\geq 80 \%$ of reported AFP cases. ${ }^{\S}$ In 2015 , both performance indicators were met nationally in six (75\%; Afghanistan, Guinea, Myanmar, Nigeria, Pakistan, and Ukraine) of the eight countries reporting WPV and cVDPV cases during 2015-2016. Although Afghanistan and Pakistan both met these AFP surveillance indicators, evidence suggests ongoing gaps in AFP surveillance quality, based on review of case epidemiology, results of environmental sampling, and subnational indicators (8).

\footnotetext{
$\$$ Adequate stool specimens require two stool specimens collected $\geq 24$ hours apart, within 14 days of paralysis onset, with arrival at a WHO-accredited laboratory in good condition.
}

TABLE 1. Number of supplementary immunization activities (SIAs) conducted and number of oral poliovirus vaccine (OPV) doses administered, by World Health Organization (WHO) region - worldwide, 2014-2015

\begin{tabular}{lrrrrr}
\hline & \multicolumn{2}{c}{2014} & & \multicolumn{2}{c}{2015} \\
\cline { 2 - 3 } \cline { 5 - 6 } WHO region & SIAs & OPV doses & & SIAs & OPV doses \\
\hline African & 142 & $775,972,255$ & & 117 & $766,000,000$ \\
Region of the Americas & 0 & 0 & 0 & 0 \\
Eastern Mediterranean & 183 & $639,908,596$ & & 101 & $495,000,000$ \\
European & 8 & $6,351,137$ & & 3 & $8,000,000$ \\
South-East Asia & 6 & $800,605,667$ & & 8 & $756,000,000$ \\
Western Pacific & 2 & $32,827,615$ & & 2 & 210,000 \\
Overall & 341 & $\mathbf{2 , 2 5 5 , 6 5 5 , 2 7 0}$ & & 231 & $\mathbf{2 , 0 2 5 , 2 1 0 , 0 0 0}$ \\
\hline
\end{tabular}




\section{Reported Poliovirus Cases}

Countries reporting WPV cases. In 2015, a total of 74 WPV cases were identified (Figure); 54 (73\%) were detected in Pakistan, and 20 (27\%) were detected in Afghanistan. No WPV cases were identified in countries outside of Pakistan and Afghanistan during 2015-2016 (to date). During January 1May 4, 2016, the low transmission season for polio, 12 cases were reported worldwide; eight were detected in Pakistan and four in Afghanistan (Table 2).

Afghanistan reported 20 cases in 16 districts in 2015, compared with 28 cases in 19 districts in 2014, representing a 29\% reduction in the number of cases reported. In 2015, $40 \%$ of cases were reported from Nangarhar province in the eastern region. During January 1-May 4, 2016, four WPV cases were detected (three in Kunar province in the eastern region and one in Helmand province), compared with one case detected during the same period in 2015.

Pakistan reported an $82 \%$ decrease in the number of WPV cases reported, from 306 cases in 44 districts in 2014 to 54 cases in 23 districts in 2015. During January 1-May 4, 2016, eight WPV cases were reported, compared with 22 cases during the same time period in 2015 , representing a $64 \%$ decrease. All five regions reporting WPV in Pakistan reported a decreased number of cases in 2015; the decrease was largest (91\% reduction in cases) in the Federally Administered Tribal Areas.
TABLE 2. Number of reported poliovirus cases, by country worldwide, January 1, 2014-May 4, 2016

\begin{tabular}{|c|c|c|c|c|c|c|}
\hline \multirow[b]{2}{*}{ Country } & \multicolumn{2}{|c|}{$\begin{array}{c}2014 \\
\text { (January-December) }\end{array}$} & \multicolumn{2}{|c|}{$\begin{array}{c}2015 \\
\text { (January 1-May 4) } \\
\end{array}$} & \multicolumn{2}{|c|}{$\begin{array}{c}2016 \\
\text { (January 1-May 4) }\end{array}$} \\
\hline & WPV & cVDPV & WPV & cVDPV & WPV & cVDPV \\
\hline
\end{tabular}

\begin{tabular}{lrrrrrr}
\hline \multicolumn{7}{l}{ Countries with endemic polio } \\
Afghanistan & 20 & 0 & 1 & 0 & 4 & 0 \\
Pakistan & 54 & 2 & 22 & 1 & 8 & 0 \\
Total & 74 & 2 & 23 & 1 & 12 & 0
\end{tabular}

Other countries with reported cVDPV cases

$\begin{array}{llrllll}\text { Guinea } & 0 & 7 & 0 & 0 & 0 & 0 \\ \text { Laos } & 0 & 8 & 0 & 0 & 0 & 3 \\ \text { Madagascar } & 0 & 10 & 0 & 0 & 0 & 0 \\ \text { Myanmar } & 0 & 2 & 0 & 0 & 0 & 0 \\ \text { Nigeria } & 0 & 1 & 0 & 0 & 0 & 0 \\ \text { Ukraine } & 0 & 2 & 0 & 0 & 0 & 0 \\ \text { Total } & 0 & 30 & 0 & 0 & 0 & 3 \\ \text { Overall } & 74 & 32 & 23 & 1 & 12 & 3\end{array}$

Abbreviations: $\mathrm{cVDPV}=$ circulating vaccine-derived poliovirus; WPV = wild poliovirus.

Countries reporting cVDPV cases. In 2015, a total of $32 \mathrm{cVDPV}$ cases were reported from seven countries. Outbreaks of cVDPV type 1 (cVDPV1) occurred in Laos (eight cases), Madagascar (10 cases), and Ukraine (two cases), and outbreaks of cVDPV type 2 (cVDPV2) occurred in Guinea (seven cases), Myanmar (two cases), Nigeria (one case), and Pakistan (two cases).

FIGURE. Number of cases of wild poliovirus worldwide — January 1, 2014-May 4, 2016*

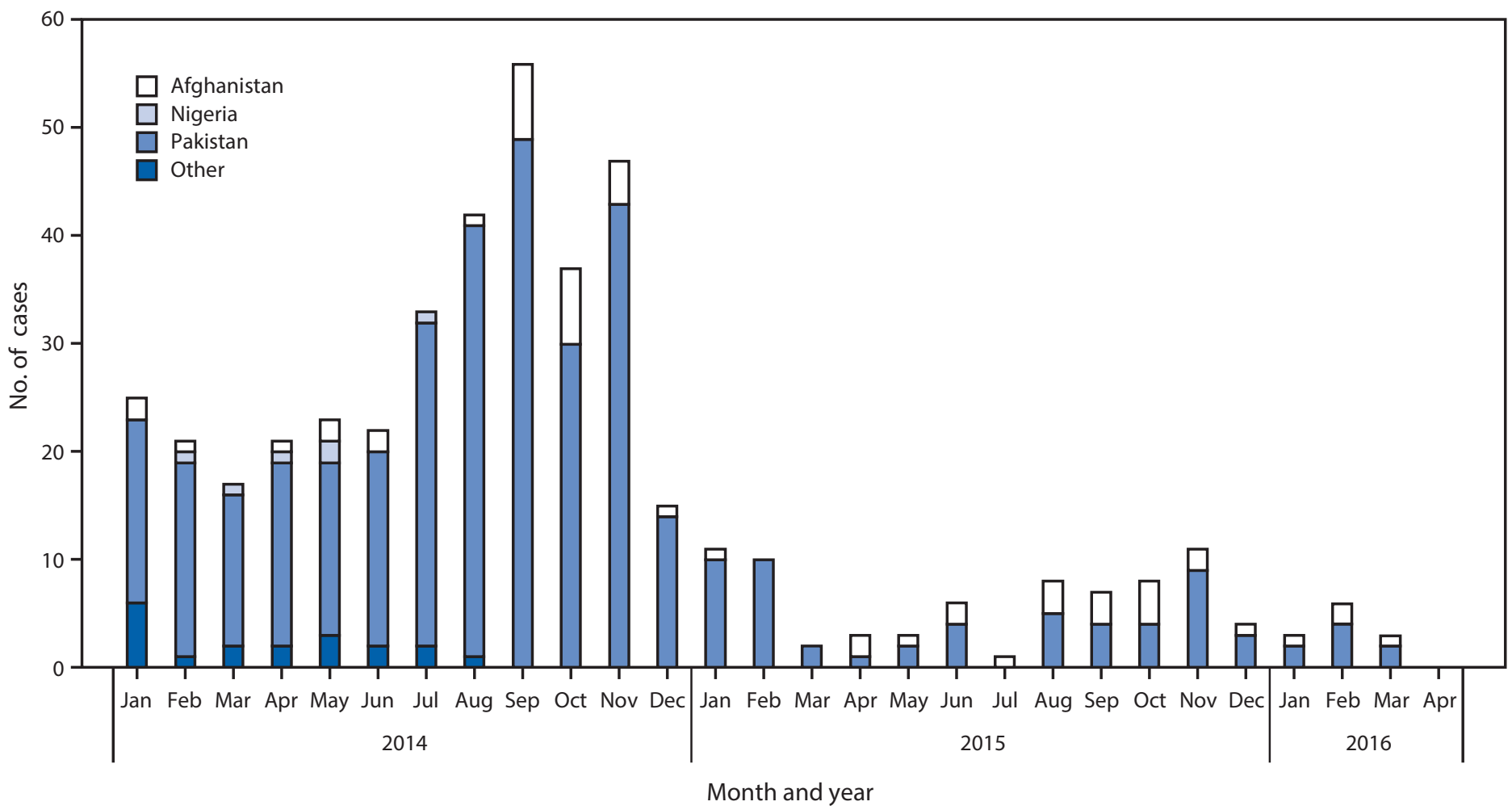

* Other countries include Cameroon ( $(=5)$, Equatorial Guinea $(n=5)$, Ethiopia $(n=1)$, Iraq $(n=2)$, Somalia $(n=5)$, and Syria $(n=1)$. 
Four of the seven countries with cVDPV cases in 2015 had continued transmission from cVDPV cases in 2014, including Guinea (one case), Madagascar (one case), Nigeria (30 cases), and Pakistan (21 cases). Countries reporting cVDPV cases in 2015 with $\geq 6$ months since the most recent case include Pakistan (most recent case February 9, 2015), Ukraine (July 7, 2015), Madagascar (August 22, 2015), and Myanmar (October 5, 2015). Laos reported three additional cVDPV1 cases in 2016 (to date), for a total of 11 cases during the outbreak; to date, no cVDPV2 cases have been reported in 2016. However, an environmental sample collected in Borno State, Nigeria, in March 2016 recently tested positive for cVDPV2 and is linked to prior circulation.

\section{Discussion}

Substantial gains toward polio eradication were made in 2015 , with a $79 \%$ decrease in the number of polio cases reported worldwide compared with the number of cases reported in 2014. The removal of Nigeria from the list of countries with endemic polio in 2015 creates the opportunity for the African Region to join the Region of the Americas and the South-East Asia, Western Pacific, and European regions, as the fifth of six WHO regions to be certified free of indigenous WPV. Certification will occur after a minimum of 3 years of sensitive AFP surveillance. In addition, the Global Commission for the Certification of Poliomyelitis Eradication's declaration of the eradication of WPV type 2 in 2015, and the absence of reported circulation of WPV type 3 since 2012, allows focus on WPV type 1 as the sole circulating type of WPV in the world, endemic only in Afghanistan and Pakistan. WHO considers the continued transmission of WPV type 1 between both countries to constitute a public health emergency of international concern under the 2005 International Health Regulations.9 Continued focus on identifying groups of children who missed polio vaccination through routine immunization or SIAs, improving SIA quality, and increasing AFP surveillance sensitivity in these countries is needed to stop transmission.

In 2015, Afghanistan had a major reduction in WPV cases. The majority of cases were reported from Nangarhar province in eastern Afghanistan, which borders Pakistan, and were genetically linked to cases in Pakistan, emphasizing the need for continued improvement of cross-border coordination and SIA synchronization. Although some children are missed during SIAs in Afghanistan because of inaccessibility and security concerns, the majority are missed during SIAs because of managerial issues, including inadequate microplanning and campaign implementation. The southern region, although accessible for program implementation, has very limited access for supervision and

\footnotetext{
http://www.who.int/mediacentre/news/statements/2016/8th-IHR-emergencycommittee-polio/en/.
}

\section{Summary}

What is already known about this topic?

Wild poliovirus (WPV) transmission is now endemic in only Afghanistan and Pakistan. During 2014-2015, outbreaks of WPV in five countries without endemic polio were successfully ended, and Nigeria was removed from the list of countries with endemic polio transmission.

What is added by this report?

WPV transmission has continued in Afghanistan and Pakistan during 2016; compared with transmission in 2015, the number of WPV cases decreased. Circulating vaccine-derived poliovirus remains a risk in areas with low oral poliovirus vaccine (OPV) coverage, with five countries reporting ongoing outbreaks during 2015-2016. In April 2016, 154 countries and territories discontinued use of type 2 Sabin vaccine by simultaneously switching from trivalent OPV (containing types 1, 2, and 3) to bivalent OPV (containing types 1 and 3 ) for routine and supplementary immunization.

What are the implications for public health practice?

With progress made toward interruption of WPV transmission in Afghanistan and Pakistan, the world is closer than ever to the eradication of polio. To stop transmission, continued cooperation between the two countries is needed, with a focus on identifying groups of missed children, improving quality of supplementary immunization activities, and increasing the sensitivity of acute flaccid paralysis surveillance.

monitoring. Innovative approaches, such as the 4th-day revisit strategy during campaigns, the use of permanent vaccination teams dedicated to regular house-to-house visits, and vaccination at transit points leading in and out of insecure areas need to continue to be regularly used to reach all missed children (5). The recent establishment of emergency operations centers at the national level and in three critical regions enhances the country's capacity to plan and implement polio eradication activities.

Progress in Pakistan accounted for most of the sharp decline in the number of polio cases during 2015-2016. The substantial gains made are, at least in part, attributable to the establishment of a cohesive national emergency operations center that implemented a rigorous National Polio Eradication Emergency Action Plan (6). However, operational problems with vaccination of all children during SIAs, program accountability at all levels, and ongoing movement of unvaccinated children across the Afghanistan-Pakistan border remain challenges facing the polio program in Pakistan.

Although no WPV cases were detected in countries without endemic WPV circulation, seven countries reported cVDPV outbreaks during 2015-2016, demonstrating the risk for VDPV emergence associated with low OPV coverage. In each of these countries, certain factors, such as the concurrent Ebola epidemic in Guinea and instability in vaccine procurement 
and public trust in Ukraine, diminished the quality of routine immunization services and allowed the emergence and spread of the outbreaks. Approximately $95 \%$ of cVDPV cases since 2006 have been caused by cVDPV2 (9). Therefore, with certification of the eradication of WPV type 2, in April 2016, 154 of 155 planned countries and territories** discontinued use of type 2 Sabin vaccine by switching from tOPV to bOPV for routine and supplementary immunization during a globally synchronized initiative that spanned 2 weeks, from April 17-May 1, 2016 (9). The global switch from tOPV to bOPV will markedly reduce the risk associated with type 2 cVDPV emergence and transmission; however, the global community must continue to support strong routine immunization service delivery to curb the risk for type 1 or type 3 cVDPV outbreaks or transmission after WPV importation from countries with endemic poliovirus transmission.

With progress made during 2015-2016 toward interruption of WPV transmission in Afghanistan and Pakistan, the world is closer than ever to the eradication of polio. Continued cooperation between the two countries is needed for this goal to be reached. In addition, the greater worldwide community needs to remain vigilant in implementing the Global Polio Eradication Initiative's Polio Eradication and Endgame Strategic Plan for 2013-2018 to end WPV and VDPV transmission (10).

\footnotetext{
** http://www.polioeradication.org/mediaroom/newsstories/The-Global-SwitchAs-It-Happens/tabid/526/news/1373/Default.aspx.
}

\section{Acknowledgments}

World Health Organization (WHO) Regional Office for the Eastern Mediterranean Region, Amman, Jordan; WHO Regional Office for Africa, Brazzaville, Congo; WHO Regional Office for Europe, Copenhagen, Denmark; WHO Regional Office for the Western Pacific, Manila, Philippines; WHO Regional Office for South-East Asia, New Delhi, India; Global Polio Laboratory Network, Geneva, Switzerland; Division of Viral Diseases, National Center for Immunization and Respiratory Diseases, CDC.
${ }^{1}$ Epidemic Intelligence Service, CDC; ${ }^{2}$ Global Immunization Division, Center for Global Health, CDC; 3 Polio Eradication Department, World Health Organization.

Corresponding author: Michelle Morales, MMorales7@cdc.gov, 404-372-6174.

\section{References}

1. Kew OM, Cochi SL, Jafari HS, et al. Possible eradication of wild poliovirus type 3-worldwide, 2012. MMWR Morb Mortal Wkly Rep 2014;63:1031-3.

2. Hagan JE, Wassilak SGF, Craig AS, et al.. Progress toward polio eradication-worldwide, 2014-2015. MMWR Morb Mortal Wkly Rep 2015;64:527-31.

3. World Health Organization. Global Polio Eradication Initiative. Polio this week as of 04 May 2016. Geneva, Switzerland: World Health Organization; 2016. http://www.polioeradication.org/Dataandmonitoring/Poliothisweek.aspx

4. Morales M, Nnadi CD, Tangermann RH, Wassilak SGF. Notes from the field: circulating vaccine-derived poliovirus outbreaks-five countries, 2014-2015. MMWR Morb Mortal Wkly Rep 2016;65:1289. http://dx.doi.org/10.15585/mmwr.mm6505a5

5. Mbaeyi C, Saatcioglu A, Tangermann RH, Hadler S, Ehrhardt D. Progress toward poliomyelitis eradication-Afghanistan, January 2014 August 2015. MMWR Morb Mortal Wkly Rep 2015;64:1166-70. http://dx.doi.org/10.15585/mmwr.mm6441a2

6. Farag NH, Wadood MZ, Safdar RM, et al. Progress toward poliomyelitis eradication-Pakistan, January 2014-September 2015. MMWR Morb Mortal Wkly Rep 2015;64:1271-5. http://dx.doi.org/10.15585/mmwr. mm6445a4

7. World Health Organization. WHO vaccine-preventable diseases: monitoring system 2015 global summary. Geneva, Switzerland: World Health Organization; 2016. http://apps.who.int/immunization_ monitoring/globalsummary/wucoveragecountrylist.html

8. Snider CJ, Diop OM, Burns CC, Tangermann RH, Wassilak SG. Surveillance systems to track progress toward polio eradicationworldwide, 2014-2015. MMWR Morb Mortal Wkly Rep 2016;65:34651. http://dx.doi.org/10.15585/mmwr.mm6513a3

9. Immunization Systems Management Group of the Global Polio Eradication Initiative. Introduction of inactivated poliovirus vaccine and switch from trivalent to bivalent oral poliovirus vaccine-worldwide, 2013-2016. MMWR Morb Mortal Wkly Rep 2015;64:699-702.

10. Global Polio Eradication Initiative. Polio eradication and endgame strategic plan 2013-2018. Geneva, Switzerland: World Health Organization, Global Polio Eradication Initiative; 2013. http://www. polioeradication.org/Portals/0/Document/Resources/StrategyWork/ PEESP_EN_US.pdf 